
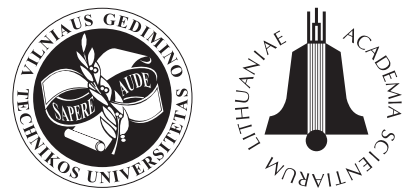

TRANSPORT

2009

24(2): 129-134

\title{
RESEARCH ON ADVANCED TECHNOLOGIES AND THEIR EFFICIENCY IN THE PROCESS OF INTERACTIONS BETWEEN DIFFERENT TRANSPORT MODES IN THE TERMINAL
}

\author{
Nijolè Batarliené $\dot{e}^{1}$ Aldona Jarašūniené $\dot{e}^{2}$ \\ Vilnius Gediminas Technical University, Plytinès g. 27, LT-10105 Vilnius, Lithuania \\ ${ }^{1}$ Dept of Transport Management; ${ }^{2}$ Transport Research Institute \\ E-mails: ${ }^{1}$ nijbat@ti.vgtu.lt; ${ }^{2}$ aldonaj@ti.vgtu.lt
}

Received 19 March 2008; accepted 10 April 2009

\begin{abstract}
The infrastructure of transport means has to be modernized and organized in such a way that operators could employ effective transportation technologies and processes and that the sustainable development of different transport modes and their proper interoperability with the transport systems of the neighbouring states are achieved. The long-term development strategy of the Lithuanian transport system notes that the solution to the problems of transport technology is based on improvements in technological supply and the rational use of the informational modeling methodology of the whole transportation process. The interaction between different transport modes in the terminal is a complex and multiplex task. To reach a successful solution for this task, a thorough evaluation and analysis of numerous factors is required and a preliminary research of respective elements is necessary. The application of advanced technologies is essential in the terminal where several road transport modes are involved in the process of interaction. Therefore, an information transmission centre (ITC) is necessary.
\end{abstract}

Keywords: advanced technologies, transport means, information infrastructure, management, terminal.

\section{Introduction}

Jarašūnienè and Vasilis Vasiliauskas (2005) note that the analysis of technological processes and their optimization in transport terminals enables the successful functioning of transport, thus guaranteeing consignee reliability, independency and quality.

Gromule, Jurshevich and Yatskiv (2006) suggest that only modern transport may reliably perform cargo and especially freight transportation and ensure the efficiency and total safety of the economic process.

Baublys (2007) emphasizes that the terminal plays an important role in freight transportation.

Batarlienè (2007) states that information technologies provide us with the new possibilities of organizing transport work and a physical location of transport means. These issues are more widely investigated by Lithuanian scientists. Advanced technologies can be used for the electronic data registration of transport means and cargo.

\section{Estimating the Advanced Transport Technologies Approach to the Long Term Development Strategy of the Lithuanian Transport System}

The major goal of transport policy pursued by the European Union (hereinafter - the EU) is creating the un- limited unified transport market providing equal conditions for its members to compete with each other. However, solutions made in the field of national transport are often ineffective because of lack of information about transport mobility, logistic requirements or unforeseen changes in policy.

Batarliene and Baublys (2007) agree that decisionmaking requires the access to reliable information and statistical data on the transport system as well as on the environment and major influencing factors.

The National Long-Term Transport and Transit Development Strategy (Long-term ... 2005) explicitly formulates only the strategic goals and measures of developing individual transport modes, whereas one of the key directions of the transport policy of the EU and Lithuania is to strengthen the interoperability of transport modes.

Road, railway, seaport and airport infrastructure has to be modernized and developed in a coordinated way, so that operators could employ effective transportation technologies and processes and promote the sustainable development of different transport modes and their proper interoperability with the transport systems of the neighboring states.

A crucial point in the development of advanced transport technologies is to accelerate the integration 
of the Lithuanian transport system into the economic community of Europe and other countries worldwide. Thus, it is necessary to create the information infrastructure of the Lithuanian transport system that could optimally function both internally and externally.

The information infrastructure of the transport system is understood as an integrated aggregate of informatics and communication means, standards, technical regulations and organizational procedures and enables the electronic accumulation of the major information resources of the transport system, their processing in a coordinated way and, with the help of computer communication means, the immediate provision of the reliable summarized information of different nature, form and purpose which is necessary for technological activities of companies, comprehensive accounting as well as for adopting the decisions of the authorities of companies or a transport system.

Creating information infrastructure is understood not as designing one huge computerized information system but as building the environment for the functioning of the information systems of companies.

The purpose of the information infrastructure of the transport system is the efficient and optimal informational maintenance of the functioning of the Lithuanian transport system. Such information infrastructure will allow:

- the acceleration and optimization of the movement of material and information flows through the computerization and informatisation of the functioning of its elements that control the above-mentioned movement;

- the integration of the Lithuanian transport system into the European transport network;

- integration into the European transport service market.

To create the information infrastructure of the transport system, it is necessary to take account of general European requirements and multilateral agreements with the neighboring countries.

The following measures in the field of developing advanced transport technologies should be mentioned:

1. Developing and introducing a transport network system of traffic and user information management and control in order to optimize the use of the infrastructure;

2. Modernizing railway transport data transmission and traffic management system and assuring its compatibility with those of the neighboring countries;

3. Drafting a programme for transport system management and rearranging the information technologies and telecommunication structure of individual transport branches for the purposes of developing intelligent transport systems while integrating the Lithuanian transport system into the EU transport information technologies and telecommunication systems;

4. Developing and introducing a computerized system that enables automatic control over the technical state of road transport, drivers' work and rest schedule as well as over information on freight being transported.

5. Introducing an integrated information system that would embrace all activities of the railway sector and assure an effective management of the total railway transport system. The process of introducing such system could be followed by the rational use of the available system capacity launching freight flows across the territory of Lithuania at the lowest cost and in the shortest period of time, the rational planning and implementation of the maintenance and repairs of the mobile and stationary objects of the railway transport system, its connection with the systems of the information technologies of other transport modes and its organic integration into the information system of all transport sectors in Lithuania.

\section{Research on the Efficiency of Information Transmission Centers (ITC)}

Adamko and Klima (2007) and Adamko, Kavička, and Klima (2005) maintain that railway service terminals are the places of a railway network equipped with costly technology based on high complexity technological procedures demanding a high degree of coordination and control skills. Ivaldi and Mccullough (2008) state that great effort is therefore exerted to find an optimal configuration of infrastructure to extensively exploit technical and human resources and to effectively organize technological procedures.

The interaction between different transport modes in the terminal is a complex and multiplex task. To reach a successful solution for this task, a thorough evaluation and analysis of numerous factors is required and a preliminary research of respective elements is necessary. The application of advanced technologies is essential in the terminal where several road transport modes are involved in the process of interaction. Therefore, an information transmission centre is necessary.

The synthesis of the structure of Information Transmission Centre (ITC). In designing ITC, the following structures such as organizational, functional, informative, technical management means etc. should be distinguished.

The synthesis of ITC structure has the following notation:

$P$ - the set of ITC or its elements possible to be made by the principle $\pi \in P$ (the possible principles of ITC functioning are usually set during its synthesis and selection of certain principles from the set $P$ ); $F$ - the set of interacting functions performed by the centre (for each principle of the centre the $p$ set is corresponding any set of functions $F(\pi)$ from which in the process of designing ITC, it is necessary to chose the sub-set $f \in F(\pi)$ sufficient for the realization of the selected principles $\pi$ ); $A$ - the set of possible elements interrelated in ITC (similar elements include ITC subdivisions, tech- 
nical means of management, means of servicing different transport modes, groups of people etc.).

Baublys (2007) accepts it will also introduce an operation of reflecting $M$ elements of the $F$ set to the set of $A$ elements. Optimum reflection should guarantee the necessary characteristics of ITC functioning and the extreme of any functioning.

In general, the task for the synthesis of ITC optimum structure should be as follows:

$$
\pi \in P,
$$

$$
f \in F(\pi),
$$

$$
\bar{A} \in A,
$$

$$
[f \in F(\pi)] M[\bar{A} \in A] \text {. }
$$

If ITC structuring principles are set, the syntheses of the optimum structure should define (1)-(4), whereas if ITC structuring principles are set and its functions are performed, then (3)-(4) are determined; furthermore, if ITC principles are set, its functions and ITC elements are performed and then (4) is defined. This is a rational reflection of the set of the performed functions to the set of ITC interrelated elements.

The task of ITC analysis consists of indicating its characteristics under the set conditions of (1)-(4).

The synthesis of the functional structure. The synthesis of ITC functional structure envisages the distribution of management operations between different transport modes.

While analysing the management process at ITC, it is important to detect elementary operation that will call separate operations elementary if their further particularisation has no sense within the limits of the synthesis of this structure. According to Hickman (2001), depending on the level of the analysed ITC, the elementary operations are those of the technological process of the terminal, financing, processing information, decision-making etc.

In general, for each elementary operation $l_{i}$ it will correspondingly show the vectors of entry $x_{i}=\left(x_{1 i}, \ldots\right)$ $\left.x_{m i}\right)$ and exit $y_{i}=\left(y_{1 i}, \ldots, y_{n i}\right)$. In most cases, the transformation of vector $x_{i}$ to vector $y_{i}$ can be noted in the following way:

$$
y_{i}=f_{i}\left(x_{i}\right),
$$

where $f_{i}$ - transformation function.

For example, as an operation can be considered a task selected according to a certain criterion of alternative $y_{i}$ in accordance with many $x_{1 i}, \ldots, x_{m i}$.

If transformation is linear, then (5) can be noted in the matrix form:

$$
y_{i}=A_{i} x_{i}
$$

where $A_{i}$ - the matrix of transformations.
The elementary operations are interrelated. The relation of the operations can be conveniently presented by the loop-less graph $G^{1}\left(E, H^{1}\right)$ the set of peaks $E$ of which corresponds to operations $l_{1}, \ldots, l_{n}$, and each ridge $h_{i j}^{1} \in H^{1}$ shows that the exit of operation $l_{i}$ is the entry of operation $l_{i}$.

As a rule, transformation (5) is related to the exploit of resources (technological capacities, finances, operational materials etc.). Then, besides logical relations between operations $l_{i}$, it is necessary to evaluate relations determined by restrictions:

$$
\phi_{k}\left[f_{i}\left(U_{i}\right)\right] \leq U_{k} ; \quad l_{i} \in E_{k} ; \quad k=\overline{1, m},
$$

where $U_{k}-$ is the amount of $k$ type resources; $E_{k} \in E$ - the sub-set of operations performing $k$ functions or using the resources of $k$ type.

Relations between operations occurring due to restrictions (7) will be called the relations of resources or functional relations that can be graphically presented after making the graph of resources $G^{2}\left(E U V, H^{2}\right)$ where the set of peaks $V=V_{1}, \ldots, V_{m}$ shows the derivation of resources (that can be fictional). Each ridge $h_{k j}^{2}$ shows that the recourses of $k$ type are necessary for operation $l_{j}$.

To obtain the total view of relations to operations, it is necessary to make joint $G\left(E \cup V, H^{1} \cup H^{2}\right)$ that should be obtained from graph $G^{1}$ by adding the resource peaks and rings of graph $G^{2}$ that will be called the graph of between-operational relations.

The segmentation of the set elements of $E$ to $N$ independent sub-sets is noted as $E_{1}, \ldots, E_{N}$, where, $E_{i} \in E$ and $E_{i} \cap E_{j}=\varnothing$ for $i, j=\overline{1, N}$ and $\bigcup_{i=1}^{N} E_{i}=E$.

The objective of the task is to obtain a segment that minimises the function, $\sum_{i=1}^{N} c\left(E_{i}\right)$, where $c\left(E_{i}\right)$ is a function defined by the set of segments $E_{i} ; i=\overline{1, N}$.

The task of the synthesis of ITC structure consists of the segmentation - solution of $E$ to a number of subsets $E_{1}, \ldots, E_{N}$ under the variety of segmentation criteria. Herewith, the proximity of operation $l_{i}$ to operation $l_{j}$ will be characterised by dimension $m_{i j}$ where $m_{i j}$ - the proportion of graph $G$ edge $h_{i j}$ gauged by the proportions of resources and relations.

The main objective of the ITC terminal is the task for managing freight handling processes. The handling process in the terminal can be formalised by a discrete mathematical model. However, given high intensity of transport flows, handling operations are performed practically incessantly.

Therefore, the handling process in the terminal can be considered as a gradually developing process in the run of time.

The model of the freight handling area of the terminal made on the basis of differential equations can be used for the analysis of freight handling by applying the theory of automatic regulation. 
Baublys (2007) admits it will analyse ITC as a linear system of automatic regulation with typical elements the stability of which can be tested by classical methods, for example, applying Noikvist-Michailov model.

Since all the elements of the system are coordinated by ITC, its separate characteristics - processing and transmission functions of incoming information should be considered while forming the management system, and therefore the coordination of the characteristics of all elements in the system is necessary. In the handling area, for every phase of entering a flow, it is necessary to identify the typical elements of the automatic management system as well as characteristics and operation principles corresponding to the mathematical models of the sector components of the objects.

The simplest mathematical model in the ITC management system is the linear differential equation with fixed coefficients. This equation links the entry of information with its exit after processing:

$$
x_{e x}(t)=x_{\text {ent. }}\left(t-t_{r}\right)
$$

its transmission function:

$$
s_{3}(p)=e^{-p t_{r}},
$$

the equation of an inert element:

$$
t_{c} x_{e x}+x_{e x}=k x_{e n t} \text {, }
$$

the transmission function:

$$
s_{4}(p)=\frac{k}{t_{p} p+1}
$$

where $k$ - the coefficient of intensifying the element of the automatic regulation system; $x_{\text {ent }}$ and $x_{\text {ex }}$. respectively the entering and exiting rates of this element.

Then, the function of the transmission of the open system:

$$
s_{0}(p)=s_{3}(p) s_{1}(p)=\frac{k e^{-p t_{r}}}{t_{p} p+1}
$$

physical-amplitude characteristics:

$$
s_{0}(j \omega)=\frac{k e^{-j \omega t_{r}}}{1+j \omega t_{p}} .
$$

If the management object is a proportional element, then, after rating (12), the transmission function of the handling area and dispatcher for the open system is:

$W(p)=s_{1}(p) s_{2}(p) s_{3}(p) s_{0}(p)=\left(1-\alpha_{0}\right)(1+\beta) \frac{k e^{-p t_{r}}}{1+p t_{p}}$,

and the characteristic of the phasic amplitude object is:

$$
W(j \omega)=\left(1-\alpha_{0}\right)(1+\beta) \frac{k e^{-j \omega t_{r}}}{1+j \omega t_{p}} .
$$

However, the handling area is a system of several elements and makes (to each other) parallel, feedback-related contours with the management organ. As known, for the system with feedback-relation:

$$
k(p)=W(p) /[1+W(p)],
$$

where $W(p)$ - is the transmission function for the open system.

The transmission function for the system of many contours closing in the management organ is the sum of the transmission functions of elements with feedback relations.

The elements of the transmission function: the wagon-storage area:

$$
k_{1}(p)=\frac{s_{1}(p) s_{0}(p)}{1+s_{1}(p) s_{0}(p)}=\frac{\left(1-\alpha_{0}\right) k e^{-p t_{r}}}{1+t_{p} p+\left(1-\alpha_{0}\right) k e^{-p t_{r}}}
$$

storage area-road transport means:

$$
k_{2}(p)=\frac{k e^{-p t_{r}}}{1+t_{p} p+k e^{-p t_{r}}},
$$

wagon-transport means:

$$
k_{3}(p)=\frac{(1+\beta) k e^{-p t_{r}}}{1+t_{p} p+(1+\beta) k e^{-p t_{r}}},
$$

the total system of the handling area:

$$
\begin{aligned}
& k(p)=\sum_{i=1}^{3} k_{i}(p)=k e^{-p t_{r}}\left[\frac{1-\alpha_{0}}{1+t_{p} p+\left(1-\alpha_{0}\right) k e^{-p t_{r}}}+\right. \\
& \left.\frac{1}{1+t_{p} p+k e^{-p t_{r}}}+\frac{1+\beta}{1+t_{p} p+(1+\beta) k e^{-p t_{r}}}\right] .
\end{aligned}
$$

Knowledge of this aspect enables us to obtain the phasic-amplitude characteristic:

$$
\begin{aligned}
& k(j \omega)=k e^{-j \omega t_{r}}\left(\frac{1-\alpha_{0}}{1+j \omega t_{p}+\left(1-\alpha_{0}\right) k e^{-j \omega t_{r}}}+\right. \\
& \left.\frac{1}{1+j \omega t_{p}+k e^{-j \omega t_{r}}}+\frac{1+\beta}{1+j \omega t_{p}+(1+\beta) k e^{-j \omega t_{r}}}\right) .
\end{aligned}
$$

Noikvist-Michailov criterion for a stable open system is formulated as follows: a closed system of automatic regulation is stable if under its open condition, the amplitude-phasic characteristic $W(j \omega)$ with frequency changing from $-\infty$ to $+\infty$ does not cover the point with coordinates $(-1, j=0)$. Testing the stability of the open system with amplitude-phasic characteristic (8) has proved that while $\omega$ is changing from $-\infty$ 
to $+\infty$, the system with feedback relation is stable. If $\omega$ is directly inserted to (8), then this proposition stands up: $W(j \omega)$ is always more than 0 . In phenomena (8) and (18), the frequency $\omega$ characterizes the periodic performance of information signals received by ITC. In managing the terminal, $\omega$ can be sufficiently extensive (hundreds of messages every day).

This linear model of operation applies to the constant fixation of freight handling processes in the terminal.

This model is also acceptable in the interactive management system. Furthermore, it serves well for describing the performance of freight stowage machine operators constantly supervising handling operation and observing the condition of technical means.

\section{Research on Vehicles Handled at the Terminal}

During the carried out research, the amount of vehicles arriving at a certain period to the terminal was registered and the duration of handling each vehicle was registered. The duration of research is 24 hours.

The vehicles were grouped in certain amounts and the handling time of each vehicle was grouped according to statistical time intervals (Fig. 1). In this case, the empirical distribution of the vehicles was reflected. If compared with the theoretic law, this shows that it corresponds to the Erlang law.

The obtained meanings have been grouped accordingly. The length of grouping intervals can be identified by the Sturges formula:

$$
\frac{\Theta_{\max }-\Theta_{\min }}{1+3.32 \lg N}
$$

where $\Theta_{\max }-$ the longest period of time, $\Theta_{\min }-$ the shortest period of time and $N$ - the amount of intervals.

The arithmetical average, dispersion, standard actual deviation of the data and distribution of the Erlang law are indicated which shows that the data can be computed according to the second-line Erlang law:

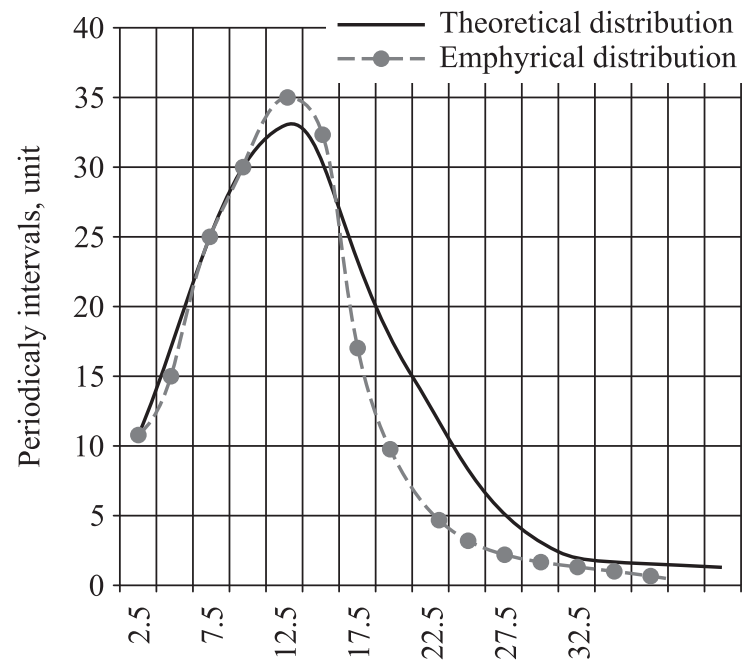

The length of grouped intervals, min

Fig. 1. Theoretical and emphyrical distributions of the vehicles

$$
f_{k}(\Theta)=v k P(k-1 ; v k \Theta) \text {. }
$$

The density of the probability of $i$ interval can be identified according to the formula:

$$
f_{k}\left(\Theta_{i}\right)=v k P\left(k-1 ; v k \Theta_{i}\right),
$$

and the theoretic frequency can be identified by:

$$
N_{\tau i}=f_{k}\left(\Theta_{i}\right) \Delta \Theta_{i}
$$

The distribution of the duration of vehicle handling $P\left(k-1 ; v k \Theta_{i}\right)$ is identified by the table of the Poisons law, for $v k \Theta_{i}$ meaning the linear interpolation has been used.

The example of the sequence of computing shows the purpose of using the second-line Erlang law for grouping the first interval of approximation:

1. $v k \Theta_{i}$; has been identified;

2. It has been identified according to the table of the Poisons law:

$$
P\left(k-1 ; v k \Theta_{i}\right) \text {; }
$$

3. The density of probability in the middle of each group has been identified:

$$
f_{2}(\Theta)=P\left(k-1 ; v k \Theta_{i}\right) \text {; }
$$

4. Probability for each interval of grouping has been identified according to the formula:

$$
\Delta \Theta_{i} f_{2}\left(\Theta_{i}\right)
$$

where is the quantity of interval $\Delta \Theta$ for each $i$-th group;

5. The identified theoretic frequencies:

$$
N_{\tau i}=\left(\sum N_{i}\right)\left[\Delta \Theta_{i} f_{2}\left(\Theta_{i}\right)\right],
$$

where $\sum N_{i}=N$.

However, this quantity also may not coincide with a general actual amount of measurements.

Such difference can be obtained by computing and rounding down the whole numbers:

$$
\sum N_{\tau i}, \Delta \Theta_{i} f_{2}\left(\Theta_{i}\right)
$$

The results of computation are presented in the table where the approximation by applying the third-line Erlang law is also introduced. The meaning $x^{2}$ computed for the third-line law is much lesser if compared with that computed by the second-line Erlang law. Consequently, it can be concluded that the third-line Erlang law is selected:

$$
\begin{aligned}
& f_{3}(\Theta)=\frac{0.5^{3} \Theta^{2} e^{-0.5 \Theta}}{2 !}=0.0625 \Theta^{2} e^{-0.5 \Theta} \\
& f_{3}(\Theta)=\frac{0.5^{3} \Theta^{2} e^{-0.5 \Theta}}{2 !}=\frac{0.125 \Theta^{2} e^{-0.5 \Theta}}{2 !}= \\
& 0.0625 \Theta^{2} e^{-0.5 \Theta}
\end{aligned}
$$


The picture shows how theoretic distribution corresponds to the real researched data.

Research has demonstrated that handling vehicles in the terminal different operations in which are performed is theoretically defined by the third-line Erlang law relating to distribution.

\section{Conclusions}

1. Research on technological processes and their optimization in transport terminals enables the successful functioning of transport, thus guaranteeing consignee reliability, independency and quality.

2. The article reveals that for a successful solution to a complex and multiplex task, a thorough evaluation and analysis of numerous factors is required and a preliminary research of respective elements is necessary.

3. Applying advanced technologies is essential in the terminal where several road transport modes interact. Thus, establishing an information transmission centre is necessary.

4. The synthesis of ITC functional structure envisages the distribution of management operations between different transport modes.

5. The article explains that the model of the freight handling area of the terminal made on the basis of differential equations can be used for the analysis of freight handling applying the theory of automatic regulation.

6. Since all the elements of the system are coordinated by ITC, its separate characteristics should be considered while forming the management system. Therefore, coordinating the characteristics of all the elements of the system is necessary.

7. The analysed model is also acceptable in the interactive management system as it serves well for describing the performance of freight stowage machine operators constantly supervising handling operation and observing the condition of technical means.

8. The researched data has been processed applying the methods of mathematical statistics, and thus handling vehicles in the terminal is theoretically defined by the third-line Erlang law relating to distribution.

\section{References}

Adamko, N.; Kavička, A.; Klima, V. 2005. Towards versatile simulation models of transport nodes, Komunikácie / Communications: Scientific Letters of the University of Zilina 2: 49-53.

Adamko, N.; Klima, V. 2007. Optimisation of railway terminal design and operations using Villon generic simulation model, Transport 23(4): 335-340.

Baublys, A. 2007. Probability models for assessing transport terminal operation, Transport 22(1): 3-8.

Batarliené, N. 2007. Implementation of advanced technologies and other means in dangerous freight transportation, Transport 22(4): 290-295.

Batarliené, N.; Baublys, A. 2007. Mobile solutions in road transport, Transport 22(1): 55-60.
Gromule, V.; Jurshevich, J.; Yatskiv, I. 2006. Application of simulation modelling at the stage of transport node planning (by the example of a bus station in Pardaugava), in Proceedings of the 6th International Conference Reliability and Statistics in Transportation and Communication (Relstat'06), October 25-28, 2006, Riga, 178-186.

Hickman, M. D. 2001. An analytic stochastic model for the transit vehicle holding problem, Transportation Science 35(3): 215-237.

Ivaldi, M.; Mccullough, G. 2008. Subadditivity tests for network separation with an application to U.S. railroads, Review of Network Economics 7(1): 159-171. Available from Internet: $<$ http://www.rnejournal.com/artman2/uploads/1/ivaldi_et_ al_RNE_march08.pdf > .

Jarašūnienè, A.; Vasilis Vasiliauskas, A. 2005. Estimation of technological process in railway customs, in Trans \& $M O-$ TAUTO'05+: 23-25 November 2005, Veliko Tarnovo: Proceedings. Vol. 5, Logistics \& Management. Sofia: ScientificTechnical Union of Mechanical Engineering, 165-168.

Long-term (until 2025) development strategy of the Lithuanian transport system. 2005. Resolution No 692 of the Government of the Republic of Lithuania of 23 June 2005. Available from Internet: <http://www.i-manager.lt/upload/200612/ Strategy_EN.doc $>$. 